\title{
Diagnostic Evaluation of Ocular Toxocariasis Using High-Penetration Optical Coherence Tomography
}

\author{
Noriyasu Hashida Kei Nakai Kohji Nishida \\ Department of Ophthalmology, Osaka University Graduate School of Medicine, Osaka, \\ Japan
}

\section{Key Words}

Toxocara $\cdot$ Optical coherence tomography $\cdot$ High penetration $\cdot$ Uveitis

\begin{abstract}
Purpose: To study a case with intraretinal Toxocara larvae in a granuloma using highpenetration optical coherence tomography (HP-OCT). Methods: A 50-year-old man, who was referred to our clinic with ocular toxocariasis, initially presented with granuloma formation in the retina. We followed the treatment-associated changes in the toxocariasis lesion by a series of examinations using HP-OCT before and after a systemic corticosteroid and anthelmintic therapy. Results: The posterior pole granuloma initially appeared as a yellowish-white intraretinal lesion with an exudative focus and dye leakage seen on angiography. After therapy, the lesion was no longer exudative. During treatment-free periods, an intraretinal lesion was seen protruding from the retinal surface into the vitreous cavity. HP-OCT confirmed the elevated lesion protruding from the retinal surface into the vitreous cavity. With treatment, the protruding focus flattened during the following 8 months, but the lesion recurred after the treatment stopped. Conclusions: The findings and clinical course strongly suggested ocular toxocariasis. The protruding retinal lesion may have been an ocular manifestation of toxocariasis. HP-OCT was useful in the follow-up and diagnosis.

(c) 2014 S. Karger AG, Basel
\end{abstract}

\section{Introduction}

Toxocara canis is a nematode parasite and a possible cause of posterior and infectious uveitis. There are 2 types of toxocariasis: visceral and ocular. Ocular toxocariasis is a larva migrans that causes uveitis due to the migration of the Toxocara larvae into the eye. Ocular 
Hashida et al.: Diagnostic Evaluation of Ocular Toxocariasis Using High-Penetration Optical Coherence Tomography

manifestations are classified further according to the site of the primary foci into the posterior pole granuloma, a peripheral inflammatory mass, and endophthalmitis [1-4]. Since ocular toxocariasis does not present with clear serologic manifestations, its diagnosis is based on the results of serum immunologic examination for Toxocara antibodies by enzymelinked immunosorbent assay (ELISA) or clinical findings. Pathologically, ocular toxocariasis has been reported to cause granulation in the retina $[2,5]$. Optical coherence tomography (OCT) is useful for noninvasively examining these intraretinal lesions. Previous studies have reported using OCT to examine the chorioretinal lesions of ocular toxocariasis, but delineation of the retina and choroid was unsatisfactory because of insufficient resolution and penetrability [5-7].

High-penetration OCT (HP-OCT), a new modality with improved tissue penetrability due to the use of a wavelength $(1,050-1,060 \mathrm{~nm})$ that is longer than the conventional wavelength $(840 \mathrm{~nm})$, characteristically provides clear and continuous images from the retina to the sclera-choroidal interface [8]. In the current study, we report a case of Toxocara with a posterior pole granuloma, and we follow treatment-associated changes in the retinal lesions of toxocariasis by serial examinations using HP-OCT.

\section{Case Report}

The patient was a 50-year-old man who reported blurred vision and floaters in the right eye that persisted for about 6 months. He then consulted a local ophthalmologist. He was diagnosed with panuveitis and referred to our department. There was no previous ophthalmic history and his general health was good. He had a 10-year history of contact with dogs, but no history of ocular trauma. On presentation, the best-corrected visual acuity (BCVA) in the affected eye was 20/25, and the ocular pressure was normal. Results of slit lamp examination showed inflammatory cells $(2+)$ and infiltration in the anterior chamber of the right eye. The fundus examination showed (1+) vitreous opacities, $(2+)$ vitreous cells, phlebitis, moderate disc swelling, reddening, yellowish-white retinal lesions in the inferotemporal region of the optic disc, and a white exudative lesion near the inferior arcade (fig. 1a). The left eye was normal. Fluorescein angiography showed a hyperfluorescent lesion and dye leakage from the middle to late phases with a hyperfluorescent optic disc. Peripheral blood tests, including blood cell count, erythrocyte sedimentation test, C-reactive protein, and serum angiotensin converting enzyme, were within the normal ranges. Serum toxoplasma antibody and viral antibodies were negative. Chest X-rays were normal. No signs suggested endogenous uveitis, and no clinical findings suggested uveitis due to tuberculosis or syphilitic, fungal, or viral infection. Examination of the patient's serum for antibody to $T$. canis larvae by ELISA showed an elevated antibody level (fig. 2).

Based on characteristic funduscopic findings, a diagnosis of ocular toxocariasis was made. Systemic albendazole (ALBENZA ${ }^{\circledR}$, GlaxoSmithKline, Brentford, UK) (800 mg twice daily) was prescribed with oral steroids (initial dose $30 \mathrm{mg} /$ day), which resulted in the resolution of the vitreous opacity. However, the lesion near the arcade seemed to be white and enlarged. It was alleviated because the exudative lesion was dried out with treatments after 1 month (fig. 1b). However, 3 months after treatment initiation, the toxocariasis recurred during a treatment-free period (fig. 1c). In addition to the primary lesion, 2 other yellowish-white retinal lesions with vitreous flares appeared. Anthelmintic albendazole (ALBENZA $800 \mathrm{mg}$ twice daily) and oral steroids (initial dose $20 \mathrm{mg} /$ day) were resumed, and the infectious lesions were controlled gradually after another 2 months (fig. 1d). The BCVA in the right eye improved to 20/12.5. Two months after the retreatment was 
Hashida et al.: Diagnostic Evaluation of Ocular Toxocariasis Using High-Penetration Optical Coherence Tomography

terminated, an intraretinal lesion was observed protruding from the retinal surface into the vitreous cavity (fig. 3a). Examination of this site by HP-OCT confirmed an elevated lesion protruding from the inner retina to the vitreous cavity (fig. 3e). The lesion was in the inner retinal layer, and the retinal pigment epithelium (RPE) and choroid were intact. The anthelminthic medication (800 $\mathrm{mg}$ twice daily) was resumed, and the elevated lesion resolved on OCT within the following 8 months (fig. 3b, f). One year after the therapy, signs of inflammation and the elevated retinal lesion had disappeared along with the reduction of blood anti- Toxocara canis antibody titer (fig. 2). Unexpectedly, after 4 months, the elevated lesion reappeared (fig. 3c, g) and anthelmintic monotherapy (800 mg twice daily) was resumed, resulting in lesion control after 2 months (fig. $3 \mathrm{~d}, \mathrm{~h}$ ). The patient was subsequently treated with anthelminthic drug and steroid tapering. At the last examination after 21 months of follow-up, the BCVA was 20/12.5 with no evidence of active inflammation. The patient continues to be followed untreated without complications.

\section{Discussion}

The ocular manifestations of toxocariasis are usually vitreous opacities, solitary chorioretinal granuloma formation, and sometimes a tractional retinal detachment $[1-4,7]$. In the current study, we obtained detailed information about the retinal lesions of ocular toxocariasis by using HP-OCT. Particularly continuous images from the retina to the choroid, which used to be interrupted by the shadows of the lesions, could be examined. The lesion protruding from the inner retinal layer into the vitreous cavity underwent morphologic changes during the response to anthelmintic medication and eventually disappeared, suggesting that it was due to Toxocara. Ashton [2] reported histologic findings of tears in Bruch's membrane and RPE in posterior pole granulation-type lesions of ocular Toxocara, suggesting that Toxocara larvae invade and migrate to the retina from the choroid.

Conventional OCT was unsatisfactory regarding the penetrability of the scan laser and the poor resolution for visualizing the areas under the lesion. In the current study, an ocular toxocariasis lesion was delineated by HP-OCT with improved resolution compared to conventional OCT. The retinal structures in the lesion before and after treatment could be examined in detail. We could not provide HP-OCT images of the first visit because the patient was advised to undergo HP-OCT, but he refused. Two months after treatment termination, he experienced recurrence, and we then were able to measure and evaluate these lesions. The lesion in fig. 1 was dome-shaped; we confirmed it with fundoscopy and the domeshaped primary lesion existing in the retina was confirmed with HP-OCT in another case (online suppl. fig. 1, www.karger.com/doi/10.1159/358191). According to a previous report [5], using conventional OCT, the retinal lesions of ocular toxocariasis were thought to be diagnosed differentially from idiopathic choroidal neovascularization, probably because the view is blocked by the lesions and the conditions from the deep layer of the retina to the choroid are unclear. It is difficult to draw the images of the chorioretinal lesions of ocular toxocariasis using commercially available OCT using a scanning laser with a shorter wavelength of about $840 \mathrm{~nm}$; the inner retinal layers usually show a high reflectivity with adjacent areas of low or no reflectivity [5, 6]. HP-OCT with a long wavelength light source of about $1,050 \mathrm{~nm}$ allows visualization of the posterior choroid and sclera [8]. It was also difficult to detect chorioretinal lesions with normal devices because strong acoustic shadows masked the details; however, HP-OCT could visualize the cell layers beneath the protruding lesions in this case more clearly. OCT using enhanced depth imaging might allow the evaluation of chorioretinal lesions [9]; however, HP-OCT is considered useful for detecting 
Hashida et al.: Diagnostic Evaluation of Ocular Toxocariasis Using High-Penetration Optical Coherence Tomography

chorioretinal lesions because the border with the choroid can be identified clearly and the acoustic shadow from protruding lesions is markedly attenuated.

The OCT findings in the current patient suggested that there are 2 types of toxocariasis. One is the granulation type caused by dead Toxocara with excessive inflammation induced by an immunologic response to the dead body of the parasite [2]. The other is a type caused by living Toxocara, which protrudes into the vitreous cavity and shows no marked inflammatory reaction, as in the current patient. Our patient had a white elevated retinal lesion with vitreous opacity and inflammation at the initial examination, but the vitreous opacity gradually resolved after initiation of treatment and the lesion protruding into the vitreous cavity had unremarkable inflammation. Therefore, it is likely that inflammation is milder while the parasite is alive.

Concerning the disease with a living parasite, one study reported a lesion due to a larva seen by funduscopy migrating in the eye without formation of an inflammatory mass [10]. Sorr [11] reported a patient with ocular toxocariasis in whom a white lesion with larva continuously migrated in the retina over a time span of 4 years. These migrating lesions suggest that Toxocara larvae can survive for a long period of time in the eye and that an elevated lesion does not necessarily mean the death of the parasite. From these reports, the lesion protruding into the vitreous cavity from the inner retina is considered to have been caused by a surviving larva.

In the current study, we present a rare case with a migrating posterior pole granuloma, which suggests that a retinal lesion can develop into a protruding mass, implying another new ocular manifestation of toxocariasis. Moreover, HP-OCT was useful for noninvasive and sequential evaluation of the lesion site, severity of tissue damage, and treatment responses.

\section{Disclosure Statement}

The authors have no proprietary or commercial interest in any material discussed in this article.

\section{References}

1 Wilder HC: Nematode endophthalmitis. Trans Am Acad Ophthalmol Otolaryngol 1950;55:99-109.

2 Ashton N: Larval granulomatosis of the retina due to Toxocara. Br J Ophthalmol 1960;44:129-148.

-3 Wilkinson CP, Welch RB: Intraocular Toxocara. Am J Ophthalmol 1971;71:921-930.

4 Taylor MR: The epidemiology of ocular toxocariasis. J Helminthol 2001;75:109-118.

5 Higashide T, Akao N, Shirao E, Shirao Y: Optical coherence tomographic and angiographic findings of a case with subretinal Toxocara granuloma. Am J Ophthalmol 2003;136:188-190.

-6 Suzuki T, Joko T, Akao N, Ohashi Y: Following the migration of a Toxocara larva in the retina by optical coherence tomography and fluorescein angiography. Jpn J Ophthalmol 2005;49:159-161.

-7 Verallo 0, Fragiotta S, Verboschi F, Vingolo EM: Diagnostic aspects and retinal imaging in ocular toxocariasis: a case report from Italy. Case Rep Med 2012;2012:984512.

-8 Ikuno Y, Kawaguchi K, Nouchi T, Yasuno Y: Choroidal thickness in healthy Japanese subjects. Invest Ophthalmol Vis Sci 2010;51:2173-2176.

-9 Nakayama M, Keino H, Okada AA, Watanabe T, Taki W, Inoue M, Hirakata A: Enhanced depth imaging optical coherence tomography of the choroid in Vogt-Koyanagi-Harada disease. Retina 2012;32:2061-2069.

-10 Rubin ML, Kaufman HE, Tierney JP, Lucas HC: An intraretinal nematode (a case report). Trans Am Acad Ophthalmol Otolaryngol 1968;72:855-866.

11 Sorr EM: Meandering ocular toxocariasis. Retina 1984;4:90-96. 


\begin{tabular}{l|l}
\hline Case Rep Ophthalmol 2014;5:16-21 \\
\hline DOI: 10.1159/000358191 & $\begin{array}{l}\text { @ 2014 S. Karger AG, Basel } \\
\text { www.karger.com/cop }\end{array}$ \\
\hline
\end{tabular}

Hashida et al.: Diagnostic Evaluation of Ocular Toxocariasis Using High-Penetration Optical Coherence Tomography
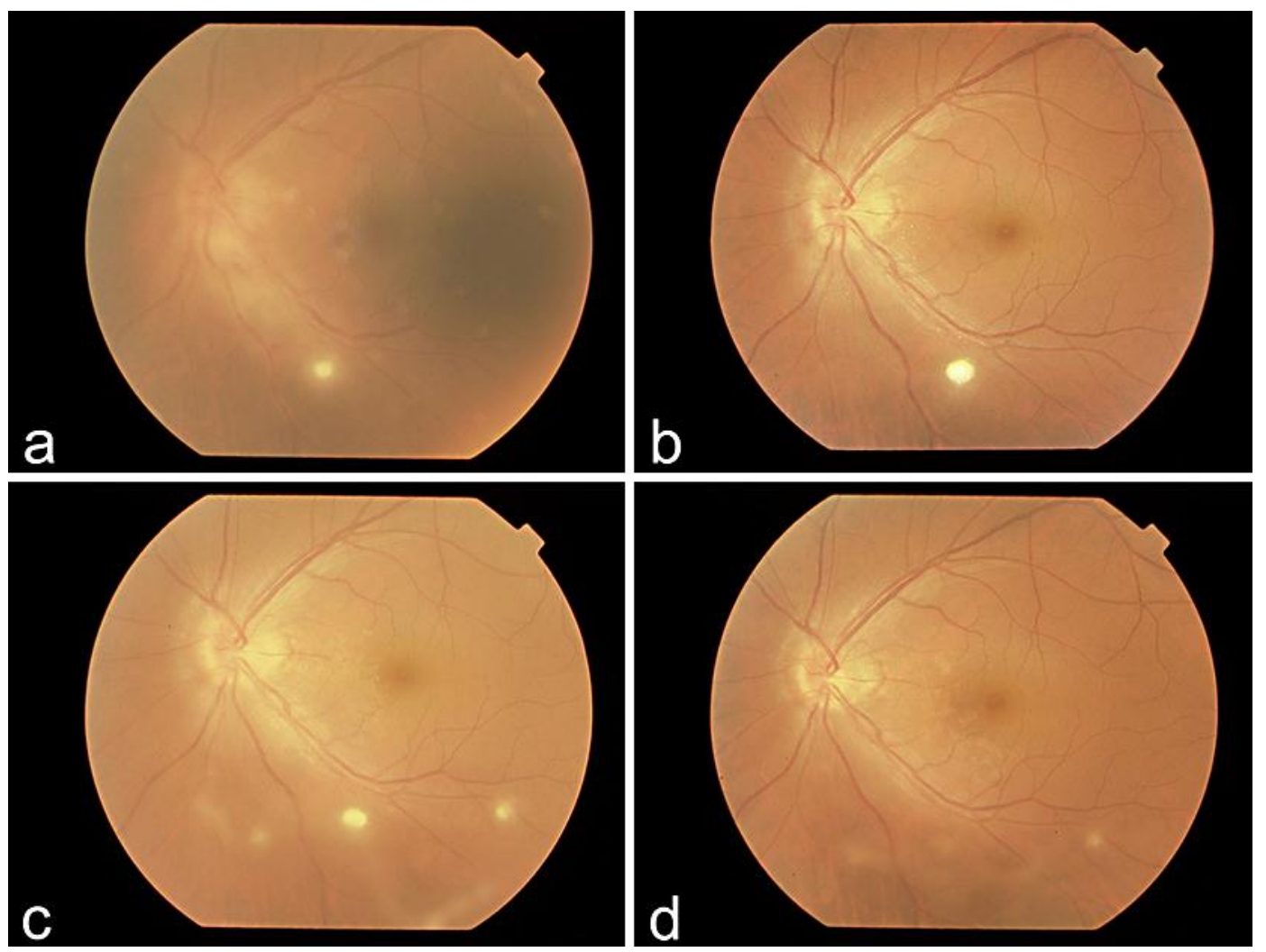

Fig. 1. A fundus photograph shows vitreous opacities and yellowish-white intraretinal lesions in the inferotemporal region of the optic disc with papilloedema at the first visit (a), resolution of the vitreous opacity and inflammatory changes at the foci 1 month after anthelmintic and steroid therapies (b), and recurrence of the primary lesion with two additional yellowish-white retinal lesions and vitreous flare 3 months after treatment (c). d These lesions were finally controlled with anthelmintic and steroid therapies after 2 months.

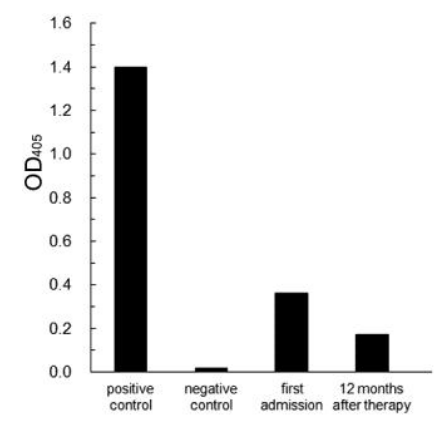

Fig. 2. Serology ELISA data for T. canis. The antibody titer deceased after therapy. OD 405: optical density at $405 \mathrm{~nm}$. 
Case Reports in

Ophthalmology

\begin{tabular}{l|l}
\hline Case Rep Ophthalmol 2014;5:16-21 \\
\hline DOI: 10.1159/000358191 & $\begin{array}{l}\text { ○ 2014 S. Karger AG, Basel } \\
\text { www.karger.com/cop }\end{array}$ \\
\hline
\end{tabular}

Hashida et al.: Diagnostic Evaluation of Ocular Toxocariasis Using High-Penetration Optical Coherence Tomography
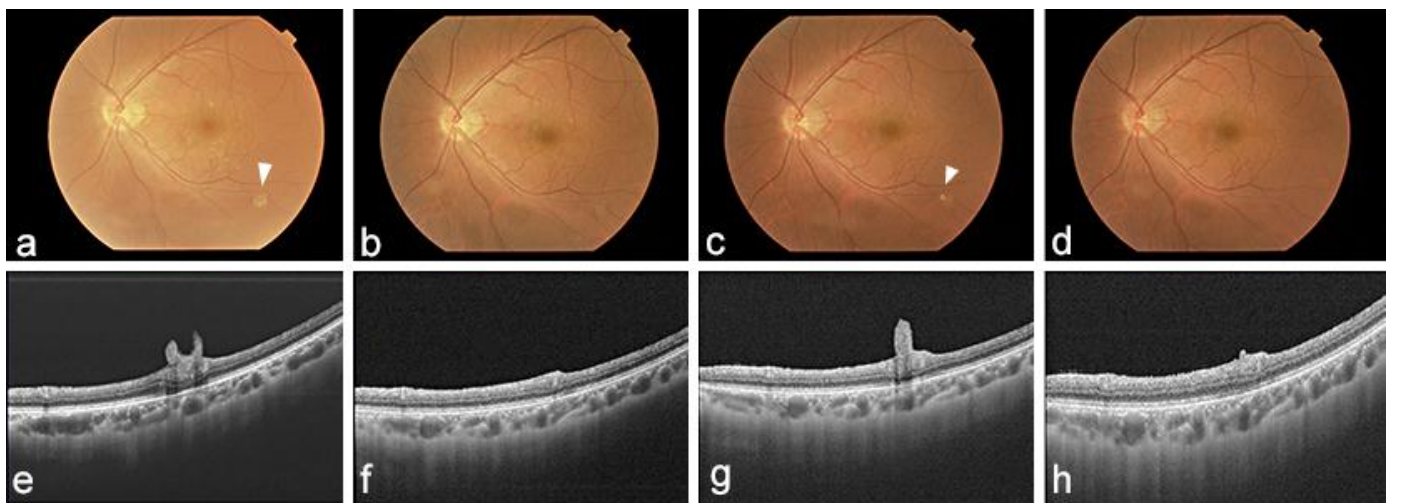

Fig. 3. A fundus photograph and optical coherence tomography images show a white intraretinal lesion (arrowhead, a) protruding from the retinal surface into the vitreous (a, e), resolution with anthelmintic and steroid therapies within the next 8 months (b, f), recurrence with a protruding retinal lesion (arrowhead, c) after 4 months (c, g), and resolution without active inflammation 2 months after anthelmintic monotherapy $(\mathbf{d}, \mathbf{h})$. 